\title{
Psicología comunitaria y expresiones psicosociales de la pobreza: contribuciones para la intervención en políticas públicas*
}

\author{
Community Psychology and Psychosocial Expressions of \\ Poverty: Contributions for Public Policy Intervention
}

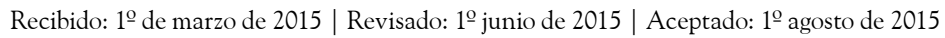

\author{
VERÔNICA MORAIS XIMENES ** \\ Elívia CamurÇa Cidade \\ BÁRbara BARbosa NEPOMUCENO \\ Universidade Federal do Ceará, Fortaleza, Brasil
}

doi:10.11144/Javeriana.up14-4.pcep

Para citar este artículo: Ximenes, V., Cidade, E., \& Nepomuceno, B. (2015). Psicología comunitaria y expresiones psicosociales de la pobreza: contribuciones para la intervención en políticas públicas. Universitas Psychologica, 14(4), 1411-1424. http:// dx.doi.org/10.11144/Javeriana.up14-4.pcep

* Artículo resultante de la investigación "Impactos de la pobreza en el desarrollo de la salud comunitaria", financiada por el Edital MCTI/CNPq/MEC/ CAPES N o 07/2011.

*** vemorais@yahoo.com.br

eliviacamurca@gmail.com

bbnepomuceno@yahoo.com.br
RES UMEN

El objetivo de este trabajo es analizar, desde la psicología comunitaria, las expresiones psicosociales de la pobreza y sus contribuciones para la intervención en políticas públicas. La psicología comunitaria critica los factores perpetuadores de aspectos materiales y simbólicos que interfieren en la constitución subjetiva de los pobres. La investigación exploratoria, de carácter cuantitativo y cualitativo, fue realizada con 417 sujetos adultos de dos comunidades de Brasil, una rural y otra urbana. La pobreza involucra explicaciones de orden moral en relación con la persona pobre. Se hace mayor referencia a la familia, a los vecinos y a la religión como fuentes de apoyo, lo cual resalta la necesidad de estrategias de fortalecimiento de las relaciones comunitarias y el redimensionamiento de la intervención en políticas públicas.

Palabras clave

psicología comunitaria; pobreza; políticas públicas; subjetividad; apoyo social.

\footnotetext{
A B S T R A C T

The purposeis to analyze, from Community Psychology's perspective, psychosocial expressions of poverty and their contributions for intervention in public policy. Community Psychology accents the critique about the factors that maintain those material and symbolic aspects that interfere with the subjective constitution of the poor. Exploratory research, quantitative and qualitative, was conducted with 417 adult subjects of a rural and urban community in Brazil. Poverty involves moral explanations about the poor. Family, neighbors and religion are emphasized as sources of support, which high lights the need for strategies then community relations and resizing of performance in public policy.

Keywords

community psychology; poverty; public policy; subjectivity; social support.
} 
La psicología comunitaria es un área de la Psicología Social de la Liberación (Góis, 2008) que pone énfasis en la comunidad y los modos de vida de los sujetos como potenciales de intervención. Parte de la crítica a la concepción individualista históricamente asociada al conocimiento en psicología (Montero, 2004). La tarea colectiva de la psicología comunitaria es actuar según los anhelos y las necesidades de la población (Montero \& Soon, 2009). Desde su origen, establece relación con las políticas públicas (Alfaro, 2013) y la expresión de la disciplina en América Latina enfatiza la necesidad de superación de las condiciones de pobreza de amplios sectores poblacionales, y toma el fortalecimiento de la participación social como importante elemento para la superación de la pobreza (Wiesenfeld \& Sánchez, 2012). Se tiene como foco el compartir experiencias entre grupos sociales comunes, con acceso precario a la vivienda, educación, salud, cultura y creación de empleo, facilitando modos colectivos de enfrentamiento de las adversidades.

En América Latina, la psicología comunitaria como campo del saber y de praxis debe actuar con vistas a la problematización del sistema neoliberal hegemónico, que acentúa la pobreza, la exclusión y el individualismo, en detrimento de los valores colectivos y solidarios (Rodríguez, 2012). Al cuestionarse las situaciones y los procesos psicosociales asociados a la calidad de vida de grupos en precariedad e iniquidad, la psicología comunitaria se preocupa por las variables que impiden o bloquean la vivencia de la democracia por la población (Wiesenfeld, 2011). Se constituye, por tanto, como un campo de saber interdisciplinar, capaz de contribuir a la ampliación de discusiones sobre la temática de la pobreza, a partir del establecimiento de un análisis multidimensional y crítico del fenómeno, en sus dimensiones psicosociales, integrando aspectos simbólicos, subjetivos y estrategias colectivas e individuales de afrontamiento (Wiesenfeld, 2011).

De acuerdo con Montero y Serrano-García (2011), al analizar la historia de la psicología comunitaria en América Latina, se encuentran dos constantes: el locus de la esfera de la vida pública y el establecimiento de una relación con el Estado (de confrontación o apoyo), como elementos in- herentes a los procesos de intervención. En Brasil, las acciones en psicología comunitaria empezaron a partir de las décadas de 1970 y 1980, bajo la influencia de movimientos sociales y reivindicaciones populares (Lane, 2002), que también se constituyeron en fuerza motriz para la redemocratización del país, después de la dictadura militar. En la década de los años 90, en América Latina, se consolida la actuación en acciones estatales vinculadas a la problemática de la pobreza y de la desigualdad social (Alfaro, 2013), fenómeno observado también en Brasil. Sobre la relación psicología y políticas públicas, Carvalho y Serrano-García (2008) destacan estas como capaces de producir una mejoría en la calidad de vida de la población, como campo en expansión de actuación de la ciencia psicológica y carente del desarrollo de investigaciones. Frente a tal escenario, la psicología comunitaria, a partir del paradigma de la liberación (Guareschi, 2009) y del desarrollo de metodologías participativas, se ha revelado como importante para los procesos de formulación, intervención y evaluación de políticas públicas, contribuyendo a la lucha por la superación de las opresiones sociales, propias de contextos marcados por la pobreza, a través de una mayor aproximación entre las políticas públicas y las problemáticas populares.

El presente estudio se fundamenta en los supuestos de que la pobreza se expresa a través de cuestiones materiales y simbólicas, interfiriendo para la constitución subjetiva de los individuos. Por consiguiente, sería la psicología comunitaria, como campo teórico, práctico y ético, el referente capaz de proporcionar ayuda para el fortalecimiento de la actuación de las políticas públicas y de las redes comunitarias de apoyo, a partir de la consideración de las expresiones subjetivas en contextos de pobreza. Ante lo expuesto, este artículo tiene como objetivo analizar, desde la óptica de la psicología comunitaria, las expresiones psicosociales de la pobreza y su contribución a la intervención en políticas públicas.

Por tanto, se hace un análisis de la pobreza cotidiana, expresada a través de variables sociodemográficas (índice de natalidad, acceso a renta, trabajo, educación, salud, ocio y cultura), así como un análisis de una dimensión subjetiva de la po- 
breza, a partir de las variables concepciones sobre pobreza y explicaciones de sus causas, reconocimiento de sí como pobre, dimensión moral de la pobreza (fe, esperanza y dignidad de la pobreza) y red de apoyo social.

\section{Psicología comunitaria, pobreza y políticas públicas en Brasil}

La pobreza se constituye como un fenómeno mundial, perpetuado a partir de procesos históricos de exclusión y desigualdad social (Demo, 2001). Se entiende tal fenómeno, a partir de una perspectiva multidimensional (Sen, 2010), en la cual se expande el espectro de su comprensión más allá de la privación de renta, desplazándose para un entendimiento de los diversos impactos que esta provoca en la vida de los sujetos, en las posibilidades de desarrollo de las potencialidades individuales y de establecimiento de una vida digna.

La pobreza es estructurada en el seno del capitalismo neoliberal, modelo socioeconómico vigente, fuertemente marcado por la globalización de la economía, minimización del Estado, recorte de los gastos con beneficios sociales, flexibilización del mercado de trabajo, privatizaciones y políticas destinadas al fortalecimiento del libre comercio (Accorssi, Scarparo, \& Guareschi, 2012). En América Latina, el Banco Mundial y Fondo Monetario Internacional han fortalecido la ideología neoliberal, regulado y formulado políticas públicas sociales (Gutiérrez, 2001). Desde la década de 1990, la reducción de la pobreza se vuelve pauta de organismos internacionales de desarrollo que definen como estrategia de intervención políticas de focalización, que en los países latinoamericanos corren el riesgo de traducirse en medidas paliativas, obstructivas de procesos de lucha por la universalización de derechos sociales (Sprandel, 2006).

Datos del Programa de las Naciones Unidas para el Desarrollo, 2014) revelan que en todo el mundo 1.2 billones de personas viven con menos de US\$ 1.25 por día; 1.5 billones viven en pobreza multidimensional, con superposición de privaciones de salud, educación y patrón de vida y 800 millones son vulnerables al riesgo de caer en la pobreza. En Brasil, actualmente, existen cerca de 16.2 millones de personas viviendo en extrema pobreza, y $59 \%$ de estos se ubican en el nordeste del país (Instituto Brasileiro de Geografia e Estatística [IBGE], 2011). Según el IBGE, la renta familiar per capita del $20 \%$ más rico es 17.8 veces mayor que la del $20 \%$ más pobre. En la búsqueda de la erradicación de la pobreza, en 2003, el gobierno federal brasileño implementó el Programa Bolsa Família (PBF) ${ }^{1}$.

De acuerdo con una evaluación realizada por Souza y Osório (2013), después de 10 años de funcionamiento del PBF, se dio una disminución de la pobreza y de la pobreza extrema, que sumadas bajaron del $23.9 \%$ al $9.6 \%$ de la población. Tales datos evidencian que en la última década se dieron importantes cambios, aunque hay mucho por hacer en lo que concierne al afrontamiento de la pobreza y de la desigualdad social, y a la afirmación del papel de las políticas públicas en este proceso.

En el escenario brasileño, la Carta Constitucional de 1988 fue el primer instrumento legal que reguló los derechos sociales, enfatizando la salud, la prevención para la seguridad social y la asistencia social como tríada de la seguridad política de bienestar brasileña. Así y todo, el reconocimiento legal de los derechos no implicó, de inmediato, su puesta en marcha, proceso típico de los países que establecieron su regulación social de forma tardía (Sposati, 2003). En los siguientes años, fue la promulgación de la Ley Orgánica de Asistencia Social, de la Política Nacional de Asistencia Social (Ministério do Desenvolvimento Social e Combate à Fome, 2004) y más recientemente el establecimiento del plan "Brasil Sem Miséria", acciones que se destacan en la lucha por la garantía de los derechos sociales.

1 El Programa Bolsa Família (PBF) es "un programa de transferencia directa de renta que beneficia familias en situación de pobreza y de extrema pobreza en todo el país. El Bolsa Família integra el Plano Brasil Sem Miséria BSM)" (BRASIL, 2004)

2 El programa "Brasil Sem Miséria" posee tres ejes estructurales: acceso a servicios (área de educación, salud, asistencia social y seguridad alimentaria), garantía de renta Bolsa Familia y Beneficio de Prestación Continuada (BPC) e inclusión productiva en los contextos rural y urbano. (Plano Brasil Sem Miséria, 2011) 
A pesar de tener como fundamento principal la constitución de políticas de protección social y no de caridad, eso no significó la implementación de principios de autonomía y de inclusión. Se sabe que, en contextos marcados por la pobreza, son las singularidades de acceso a la educación, los modos específicos de estructuración familiar y de organización de las experiencias individuales los elementos que interfieren en los modos en que los sujetos consiguen gestionar su situación (Rêgo \& Pinzani, 2013). Sin embargo, todavía son comunes los procesos de culpabilización del pobre por su situación. Estas visiones resaltan la cultura de la carencia, de la "falta de" (Torossian \& Rivero, 2013), en detrimento de miradas que deben enfocar los modos subjetivos de constitución de los sujetos en condiciones de pobreza.

Uno de los efectos de la culpabilización es el refuerzo en el imaginario social del pobre como alguien subalterno, servicial, incapaz, dependiente, ignorante y violento. Góis (2008) habla de la existencia de una "ideología de sumisión y resignación" que define las condiciones y el modo colectivo de vivir de la población pobre, y puede modelar sus condiciones de vida y actuar en su estructura psíquica. Se demuestra, de esta forma, la pobreza en sus dimensiones psicosociales, productora de fenómenos como el fatalismo (Martín-Baró, 1998), la vivencia de vergüenza y humillación (Zavaleta, 2007) y el establecimiento de una red de apoyo social de afrontamiento (Valla, 2000).

La psicología comunitaria desarrolla importantes contribuciones al análisis de la pobreza, por la lectura psicosocial que hace del modo de vida de las clases populares. Más allá de una atención a las condiciones de vida inherentes a este contexto, se dedica a los procesos culturales y simbólicos que constituyen y mantienen las realidades sociales. Resaltan los modos simbólicos de constitución de la pobreza y sus impactos en las formas de estructuración del psiquismo (Cidade, Moura Jr., \& Ximenes, 2012). En este sentido, la pobreza implica un tipo de existencia, tejido desde relaciones macro y microsociales, establecidas a partir de precondiciones estructurales, simbóli- cas, sociales y económicas. Siendo el hombre "un conjunto de relaciones sociales encarnadas en un individuo" (Vygotsky, 2000, p. 33), la vida en contextos marcados por opresiones sociales dejará marcas subjetivas e intersubjetivas.

\section{Método}

\section{Diseño y participantes}

Esta investigación de carácter exploratorio contó con análisis cuantitativo y cualitativo. El estudio se desarrolló en dos etapas. En la primera, se aplicó un cuestionario con preguntas de elección múltiple y en la segunda, se llevaron a cabo grupos focales con algunos participantes de la etapa anterior. Participaron en la investigación un número total de 417 sujetos, siendo $50.4 \%(N=210)$ habitantes de la Región de Grande Bom Jardim, comunidad urbana localizada en Fortaleza (Ceará-Brasil) y $49.6 \%$ (N $=207$ ) residentes de la comunidad rural de Canafístula, situada en el municipio de Apuiarés (CearáBrasil). Todos los participantes eran mayores de 18 años, vivían en las localidades hacía por lo menos un año y eran beneficiarios de algún programa de transferencia de renta, contabilizando $61.2 \%$ de la muestra. Entre los participantes, 117 eran hombres (28.1\%) y 299 mujeres (71.7 \%) -1 caso de missing-, la edad media de los participantes fue de 40.07 ( $\sigma$ $=17.33)$, siendo $34.9 \%(\mathrm{~N}=143)$ personas jóvenes, $54.7 \%(N=224)$ adultos y $10.3 \%(N=42)$ personas mayores.

La Región del Grande Bom Jardim está formada por los barrios Bom Jardim, Siqueira, Canindezinho, Granja Lisboa y Granja Portugal. Está localizada en el sudoeste de la capital, situada en una región que posee 541.511 habitantes, caracterizada por ser la más poblada y pobre de la ciudad, con $7.12 \%$ de personas viviendo en extrema pobreza (Instituto de Pesquisa e Estratégia Econômica do Brasil [IPECE], 2012). La comunidad de Canafístula está situada a 118 kilómetros de Fortaleza (capital del estado), y allí viven cerca de 170 familias, contabilizando poco más de mil personas. En el municipio de Apuiarés, 37.84 \% de la población rural vive en extrema pobreza (IPECE, 2014). 


\section{Instrumentos}

En la etapa cuantitativa de la investigación se aplicó un cuestionario de 37 ítems, el Cuestionario de Pobreza Multidimensional, que comprende datos sociodemográficos (ciudad, edad, sexo, religión, características residenciales, natalidad, salud, educación, trabajo, renta, acceso a políticas de transferencia de renta) e ítems sobre autodefinición en relación con pobreza/riqueza y de apoyo social. A los resultados de los cuestionarios se les aplicó un análisis estadístico descriptivo y de varianza, con ayuda del paquete estadístico Statistical Package for Social Sciences (SPSS) 18.0.

En la etapa cualitativa, se desarrollaron grupos focales con el objetivo de investigar los sentidos y los significados de los participantes sobre la pobreza y las estrategias de supervivencia emprendidas. Los datos cuantitativos se analizaron según la perspectiva del Análisis de Contenido Temático (Bardin, 2011), con ayuda del software Atlas Ti 5.2.

\section{Procedimiento}

Los cuestionarios fueron aplicados por los investigadores de forma individual (en mayoría de los casos) y colectivamente. En el primer caso, el entrevistador leía las preguntas al entrevistado y marcaba su respuesta; tal procedimiento se daba debido a la baja escolaridad del participante. En el segundo caso, los cuestionarios fueron autoaplicados en grupo bajo la orientación de un investigador. Tales procedimientos se dieron a partir de visitas domiciliares o de la citación previa con algún grupo de la comunidad para una aplicación colectiva.

En cuanto a los grupos focales, el equipo de investigación se citó anticipadamente con los participantes y organizó el lugar adecuado para su realización. Se llevó a cabo un total de cinco grupos focales: tres en la comunidad de Canafístula y dos en la comunidad de Bom Jardim, con una media de 6 a 12 participantes, y un total de 38 personas. La elección de estos sujetos se produjo de acuerdo a los siguientes criterios: haber respondido al cuestionario, contar con una renta personal de hasta
$\mathrm{R} \$ 339.00^{3}$, tener hasta 9 años de estudio, disponer del tiempo para participar en el estudio y firmar el Consentimiento Previo Libre e Informado.

\section{Aspectos éticos}

A partir de los parámetros e ítems que rigen la Resolución 466 de 2012 del Consejo Nacional de Salud de Brasil, que regula la investigación con seres humanos en el país, la investigación fue sometida al Comité de Ética en Investigación de la Universidad Federal de Ceará, siendo aprobada con los documentos CAAE: 07810512.3.0000.5054 y Parecer № 191.508. Además, se incluyeron en el estudio los participantes por medio del Consentimiento Previo Libre e Informado, y a cada participante se le explicaron los objetivos y finalidades de la investigación, la metodología aplicada, la no obligatoriedad de participación y su carácter voluntario, como también, la garantía de confidencialidad de la información y del anonimato del informante.

\section{Resultados}

Los datos que se presentan a continuación se estructuraron según las dimensiones pobreza cotidiana y aspectos subjetivos de la pobreza. La pobreza cotidiana es analizada en términos de cómo se manifiestan los datos sociodemográficos a través del acceso a la renta, al trabajo, a la educación, al ocio y a la salud. Los aspectos subjetivos integran las concepciones sobre pobreza, las explicaciones de las causas de pobreza, el reconocimiento de sí como pobre, la expresión moral de la pobreza y el afrontamiento posibilitado a través de la oferta de redes de apoyo.

\section{La pobreza cotidiana}

Sobre la situación laboral, $55.4 \%(N=232)$ informan no tener ningún tipo de trabajo remunerado, conforme a lo expresado en la Tabla 2. En relación con este dato, es importante anotar que el trabajo

3 El equivalente a medio salario mínimo de Brasil en 2013. 
TABLA 1

Dimensiones y variables del estudio

\begin{tabular}{ll}
\hline Dimensiones & Variables \\
\hline Pobreza Cotidiana & Trabajo \\
& Renta \\
Educación \\
Natalidad \\
Ocio y Cultura \\
Seguridad Pública \\
Salud \\
\hline Aspectos subjetivos de la pobreza & Concepción sobre pobreza \\
& Explicaciones de las causas de pobreza \\
& Reconocimiento de sí como pobre \\
& Expresión moral de pobreza (fe, esperanza y dignidad de \\
pobreza) & Afrontamiento - Red de apoyo \\
\hline
\end{tabular}

Fuente: elaboración propia.

informal esporádico no es reconocido como actividad laboral. Sea en el contexto urbano o rural, las configuraciones del mundo del trabajo están marcadas por el desempleo, la precarización, la pérdida de derechos sociales adquiridos y la fragilidad en las relaciones laborales. Algunos sujetos terminan por vivenciar situaciones de inseguridad financiera y pobreza. En el estudio, se observa que el $22.5 \%$ de la muestra está localizado en la franja de extrema pobreza, con renta inferior a $\mathrm{R} \$ 85.00$.

Debido a tal inseguridad, en familias donde hay sujetos pensionistas, es frecuente que estos se configuren como los principales proveedores del hogar, como se observa en el discurso que sigue: “(...) mi padre es viejecito y vive conmigo (...). Vivimos prácticamente de la ayuda de mi padre. Porque mi esposo, él es obrero, pero ya tiene una cierta edad y está enfermo" (GF2BJ).

Entre los entrevistados, el $61.2 \%(N=255)$ afirma recibir algún tipo de beneficio de transferencia de renta del gobierno, como se explicita en la Tabla 2 , que ha proporcionado a las familias los recursos básicos para su existencia, lo cual, en periodos de sequía en el contexto rural, evita la acentuación del hambre y de la ocurrencia de saqueos de alimentos. Los sujetos afirman que tales programas puedan contribuir a la apatía de los beneficiarios frente a una situación dada, pero no lo reconocen como la única causa de manutención. Al contrario, apuntan a un ciclo de perpetuación de la pobreza, íntimamente relacionado con el acceso precario a políticas públicas.

La cuestión de las familias es que ellas se habitúan a estas ayudas, pero ellas se habitúan, se acomodan, pero la necesidad existe porque esa ayuda no da para todo y eso repercute en la salud, eso repercute en la educación... me parece muy poco (...), pero aún así, las familias ya están tan necesitadas que para ellos, ellos no tienen esa visión de una salud mejor, de una educación mejor, de la cuestión del ocio continúan con necesidad y eso repercutiendo en la salud, en la educación. (GF2C) ${ }^{4}$

Además de la dimensión de la renta, las narrativas destacan la importancia del trabajo como actividad constitutiva del sujeto. Es recurrente, en el contexto rural, la relevancia de las pocas oportunidades de empleo, lo que anuncia, sobre todo para los jóvenes, la necesidad de migración al contexto urbano. En los grupos focales, se evidenció una fragilidad de políticas públicas dirigidas a la primera infancia, puesto que las madres confirman el desafío de la inexistencia de guarderías para sus niños, lo que impide que ocupen puestos de trabajo. Así se señala en el discurso de uno de los sujetos del grupo focal:

4 La sigla significa: $\mathrm{GF}=$ grupo focal, número del grupo focal y $\mathrm{C}$ $=$ Canafístula o $\mathrm{BJ}=$ Bom Jardim. 
TABLA 2

Características sociodemográficas de los participantes

\begin{tabular}{|c|c|c|}
\hline & $N$ & $\%$ \\
\hline \multicolumn{3}{|c|}{ Actualmente, ¿usted está ejerciendo algún tipo de trabajo remunerado? } \\
\hline No & 231 & 55.4 \\
\hline Sí & 183 & 43.9 \\
\hline No contesta & 3 & 0.7 \\
\hline Total & 417 & 100 \\
\hline \multicolumn{3}{|l|}{ Renta personal } \\
\hline Hasta 85.00 & 94 & 22.5 \\
\hline De 85.00 hasta 170.00 & 40 & 9.6 \\
\hline De 170.00 hasta 339.00 & 63 & 15.1 \\
\hline De 339.00 hasta 678.00 & 121 & 29.0 \\
\hline Más de 678.00 & 83 & 19.9 \\
\hline No contesta & 16 & 3.8 \\
\hline Total & 417 & 100 \\
\hline \multicolumn{3}{|l|}{ Tiempo de estudio } \\
\hline No estudió & 33 & 7.9 \\
\hline Hasta 5 años & 106 & 25.4 \\
\hline Estudió de 6 a 9 años & 69 & 16.5 \\
\hline Estudió de 10 a 11 años & 44 & 10.6 \\
\hline Estudió de 12 años o más & 160 & 38.4 \\
\hline No contesta & 5 & 1.2 \\
\hline Total & 417 & 100 \\
\hline \multicolumn{3}{|l|}{ Número de hijos } \\
\hline 0 & 140 & 33.6 \\
\hline $1-2$ & 114 & 27.3 \\
\hline $3-4$ & 75 & 18 \\
\hline $5-6$ & 28 & 6.8 \\
\hline 7 o más & 48 & 11.4 \\
\hline No contesta & 12 & 2.9 \\
\hline Total & 417 & 100 \\
\hline \multicolumn{3}{|c|}{$\begin{array}{l}\text { Número de beneficiarios de programa de transferencia de renta en la familia } \\
\text { (p. ej. Programa Bolsa Família y otros) }\end{array}$} \\
\hline No & 156 & 37.4 \\
\hline Sí & 255 & 61.2 \\
\hline No contesta & 5 & 1.2 \\
\hline Total & 417 & 100 \\
\hline \multicolumn{3}{|c|}{ Acceso a los servicios de salud } \\
\hline Nunca & 31 & 7.4 \\
\hline Pocas veces & 197 & 47.2 \\
\hline Frecuentemente & 79 & 18.9 \\
\hline Siempre & 107 & 25.7 \\
\hline No contesta & 3 & 0.7 \\
\hline Total & 417 & 100 \\
\hline
\end{tabular}

Fuente: elaboración propia 
Falta de guardería aquí, aquí que hay muchas madres que necesitan ir a trabajar y ayudar un poquito con el salario, pero no hay. No van y luego las llaman vagas, ah! esa vaga no quiere ir a trabajar, ella hasta tiene ganas, pero los hi(sic), tiene muchos hijos pequeños. (GF1BJ)

Destaca que entre los sujetos que tuvieron escolarización, apenas el 38.4 \% estudiaron 12 o más años, de acuerdo con la Tabla 2, tiempo suficiente para finalizar la enseñanza media y/o inserción en la enseñanza superior. Los relatos de los cinco grupos focales apuntan hacia el reconocimiento de la importancia de la escolarización para la superación de la pobreza y ponen en evidencia cambios ocurridos en el acceso formal a lo largo de los años y una relación entre la escolarización y el desarrollo local.

(...) ahí estudié poco tiempo, yo solo tengo el primer grado completo, pero me parece interesante y si la persona se interesaba más por los estudios (sic) porque Canafístula creció más con los estudios que ahora están pasando (...) mejoró bastante Canafístula, creció mucho y tuvo un aumento grande de la población después de esa energía. (GF3C)

La natalidad es otro elemento importante de análisis. De acuerdo a la Tabla 2, en contraposición a la visión del sentido común de que los pobres poseen un número elevado de hijos, la media total de la muestra fue de 2.6 hijos por persona $(\sigma=3.32)$. El test $t$ entre el número de hijos y el recibimiento del Programa Bolsa Família mostró una correlación significativa (de 0 ) entre las medias de las dos variables, en la que el número de hijos por familia disminuyó. Tal resultado responde a la visión de que quien es pobre y/o beneficiario de programa de trasferencia de renta tiene cada vez más hijos con la intención de obtener más recursos financieros del Gobierno.

En lo que respecta al acceso al ocio y a la cultura, entrevistados de los grupos focales reivindican mejoras. Está presente también una falta de confianza en las políticas de seguridad pública, como afirma un participante: "Tenemos (sic) que convivir con todo cerrado dentro de casa (...). Yo voy a la policía, llego y doy parte. La policía viene, iqué van a hacer ellos? Nada, solo mirar, pasar" (GF1BJ). En relación con las políticas de salud, se destaca de forma positiva la atención básica, lo que se contrapone a la calidad y el acceso descritos en los otros niveles de atención. Cuando se pregunta sobre el acceso a los servicios de salud cuando es necesario, el $54.6 \%$ de los entrevistados relatan nunca o casi nunca conseguirlo, de acuerdo con la Tabla 2. En los grupos focales, fueron planteadas reivindicaciones de mejora de las políticas de salud.

Yo pasé tres meses (...) para ver si conseguía una consulta (...) yo consulté con el doctor, el doctor me pasó los exámenes para hacer, todavía estoy en la lista de espera (...) si fuese una cosa más grave da tiempo a que la persona muera y no va a ser atendido. (GF1BJ)

Los inconvenientes en la realización de pruebas y de obtención de medicamentos, además de la falta y de la atención de los profesionales de la salud, fueron dificultades que se mencionaron. Se resalta también el impacto que los problemas de salud causan en la renta familiar, aumentando los gastos en salud y/o impidiendo que el sujeto trabaje. La población del contexto rural evidencia, todavía, la dificultad de acceso a los servicios de salud, debido a la inexistencia de transporte público.

\section{Aspectos subjetivos de la pobreza}

Cuando se indagó sobre la pobreza, los participantes plantearon definiciones asociadas a no tener acceso a los bienes y servicios y a factores morales. Describieron la pobreza como fenómeno asociado al hambre, a las restricciones en la alimentación, a la ausencia de vivienda y al endeudamiento; tal como se evidencia en el discurso que sigue:

Porque si hoy tienes alguna cosa en casa, pero tienes una cuenta grande en la tienda, eres pobre (...) porque tienes alguna cosa para comer en casa, pero (...) su incumplimiento es alto (...) eso lo considero hambre, si no tuvieses esa deuda estarías pasando hambre. (GF1C) 
Existe, también, referencia a otros indicadores, como el acceso restringido o precario a la escolarización y a los espacios de cualificación profesional, así como a las limitaciones de ocio y potencial de compra. "iAh! hija mía, la pobreza es no tener ocio. Los hijos (sic) piden una ropa, solo puedes comprar una ropa cada año, y mira" (GF2BJ).

Los sujetos consideran la pobreza un problema estructural inherente al modelo socioeconómico vigente, al desempleo, a la mala gestión y desvío de los recursos públicos por parte de los gobernantes. Según uno de los sujetos, "(...) en el capitalismo siempre va a existir el pobre y el rico” (GF1BJ). En contraposición, otro responsabiliza a los sujetos por su pobreza, atribuyéndola al hecho de "haberse acomodado" (GF1C) o no saber aprovechar las oportunidades ofrecidas. Habría, así y todo, cierta diferencia entre "la pobreza de barriga y la pobreza de espíritu" (GF2C), puesto que la segunda englobaría a los sujetos que valoran principios como el consumismo o que no poseen un adecuado proceso de escolarización.

Se preguntó a los sujetos, sobre si se reconocían como pobres, de acuerdo a la Tabla 3. Entre las respuestas, el $59 \%$ afirman ser "ni rico ni pobre". Tales datos permiten discusiones en cuanto a los criterios utilizados por las personas para evaluar sus condiciones. En los grupos focales, se identificó que situarse en un punto intermedio entre riqueza y pobreza se debía tanto al análisis de las condiciones históricas, lo que permitía el reconocimiento de mejoras en el acceso a la alimentación, a la escolarización y al consumo, como al reconocimiento de que existen valores morales que interfieren en este proceso de definición, al permitir que los sujetos se reconozcan como "rico de espíritu" (GF2BJ).
El análisis cualitativo permitió identificar una concepción moral asociada a la idea de sujeto en condiciones de pobreza como alguien que, por encima de todas las adversidades, debe actuar según valores y principios previamente establecidos. La pobreza estaría relacionada con tener dignidad, con un potencial de resistencia e integridad que es forjado por la vida en condiciones de pobreza y reivindicado para sí por los sujetos. Ser digno implica: tener fe, encontrar fuerzas para no rendirse ante las adversidades y no entrar en el mundo del crimen, ayudar al prójimo, saber convivir con los vecinos, tener alegría, valorar el lugar donde vive y la vida que tiene, reivindicar calidad en las acciones de políticas públicas, promover la unión entre los semejantes y valorar la simplicidad.

Según la afirmación de una de las participantes: "Piensa en días mejores. Somos (sic) pobres sí, pero tenemos, tengo la esperanza de vivir mejor" (GF2BJ). Tener fe remite a la esperanza de mejoras, a la comprensión de que existe un ser supremo que cuida e impulsa a la acción, que da la fuerza necesaria para romper con las adversidades, así como se aprecia en la siguiente expresión: “(...) sin Dios tú no puedes tener un rumbo en tu vida" (GF1C). La fe aparece relacionada con atributos que remiten al fatalismo, a la esperanza y al apoyo religioso. De acuerdo a la Tabla 4, el $40 \%$ de los entrevistados encuentran apoyo en la Iglesia siempre, casi siempre y frecuentemente.

En momentos de dificultad, la familia también se apunta como una referencia de apoyo, siendo mencionada por $69.7 \%$ de los participantes, conforme a la Tabla 4. El apoyo familiar se encuentra más en el contexto rural $(p=0.003)$ que en el urbano.

\section{TABLA 3}

¿Cómo se considera usted?

\begin{tabular}{lcc}
\hline & N & $\%$ \\
\hline Pobre & 154 & 36.9 \\
Ni rico ni pobre & 246 & 59 \\
Rico & 15 & 3.6 \\
No contesta & 2 & 5 \\
Total & 417 & 100 \\
\hline
\end{tabular}

Fuente: elaboración propia. 
TABLA 4

Apoyo social

\begin{tabular}{lcccccccc}
\hline & \multicolumn{2}{c}{ Iglesia } & \multicolumn{3}{c}{ Servicios Públicos (PSF, } & \multicolumn{2}{c}{ Vecinos } & \multicolumn{2}{c}{ Familia } \\
\hline & $N$ & $\%$ & $N$ & $\%$ & $N$ & $\%$ & $N$ & $\%$ \\
\hline Nunca & 137 & 32.9 & 206 & 49.4 & 130 & 31.2 & 28 & 6.7 \\
Pocas veces & 97 & 23.3 & 100 & 24 & 163 & 39.1 & 82 & 19.7 \\
Siempre o casi siempre & 99 & 23.7 & 42 & 10.1 & 63 & 15.1 & 187 & 44.8 \\
Frecuentemente & 68 & 16.3 & 50 & 12 & 44 & 10.6 & 104 & 24.9 \\
No contestado & 16 & 3.8 & 19 & 4.6 & 17 & 4.1 & 16 & 3.8 \\
Total & 417 & 100 & 417 & 100 & 417 & 100 & 417 & 100 \\
\hline
\end{tabular}

Fuente: elaboración propia

En el grupo focal, destaca que no se identificaron discursos en el contexto urbano que se remitiesen al apoyo familiar como soporte para la resolución de conflictos, al contrario de lo que ocurrió en el rural: "Para mí la familia es todo lo más precioso (...). Es donde me apoyo, cuando tengo un problema, son las primeras personas que busco" (GF2C).

Por otro lado, el $49.4 \%$ de los entrevistados reconocen el Estado (los servicios públicos) como fuente de amparo. "La Iglesia, ella ayuda mucho, en la parte del apoyo. Hasta materialmente también (...). Pero tenemos más apoyo de la religión que del Estado" (GF1BJ). El ANOVA no reveló diferencias significativas entre las comunidades urbana y rural $(p=0.598)$.

El apoyo social encontrado en las relaciones del vecindario también fue bajo, ya que el $25.7 \%$ de los sujetos afirma obtener apoyo frecuente, siempre o casi siempre. La realización del ANOVA reveló diferencias significativas entre los dos tipos de comunidad $(p=0)$, siendo mayor el apoyo del vecindario en el contexto rural.

\section{Discusión}

La realidad que se presenta de los sujetos evidencia que vivir en condiciones de pobreza implica someterse a una serie de privaciones de acceso a la educación, a los cuidados de salud, al trasporte público y a la renta. Sin embargo, destaca la expansión, en los contextos urbano y rural, del acceso a las políticas de transferencia de renta y de previsión social mediante la concesión de pensiones, posibilitando así que las familias tengan alguna ganancia y salgan de la pobreza extrema. La problemática del hambre se presenta con un nuevo traje, descrita en términos de la baja calidad nutricional, pero no la ausencia de alimentos.

El predominio del trabajo informal pone en evidencia que, a veces, las ocupaciones disponibles para los sujetos en condiciones de pobreza son aquellas que ratifican un lugar de subordinación, de vulnerabilidad al desempleo, a la baja remuneración y a las inestabilidades del mercado de trabajo (Castel, 2006). Se destaca que, en el contexto rural, las condiciones climáticas y la precariedad en el acceso a las políticas de incentivo a la producción del pequeño trabajador minimizan las posibilidades de obtener condiciones financieras para permanecer en el campo, lo que genera procesos migratorios, modificando modos tradicionales de dirigir la vida.

La articulación entre las políticas de educación y las políticas de transferencia de renta, a partir del establecimiento de condicionalidades, ha posibilitado la garantía de carácter puntual de concesión del recurso financiero, así como el aumento de la escolaridad de la población y su valoración como estrategia para la superación de la pobreza (Fernandes, 2014; Silva, Brandão, \& Dalt, 2009). Se trata de la posibilidad de superación de una deuda histórica del poder público brasileño para con su pueblo. Revelan inclusive los datos, en consonancia con otras investigaciones (Alves \& Cavenaghi, 2013), que en familias pobres se ha disminuido la fecundidad a medida que se ha presentado un aumento progresivo de los años de escolarización. 
Se suman a esto, las transformaciones resultantes de los arreglos familiares, sobre todo en lo concerniente al papel de las mujeres, que por referenciar la titularidad del recurso del programa de transferencia de renta Bolsa Família, han aumentado su poder de decisión y su autonomía en la gestión del hogar (Rêgo \& Pinzani, 2013). De esta forma, se afirma la necesidad de políticas que enfaticen "pleno empleo y trabajo decente para todos" (Alves \& Cavenaghi, 2013, p. 243) y que consideren las especificidades de las familias monoparentales femeninas, donde la mujer es la única proveedora del hogar, responsable por el trabajo externo, actividades domésticas y cuidado de los hijos (Camargo, Curralero, Lício, \& Mostafa, 2013). En este sentido, la ausencia de guarderías, y no una posible acomodación de las madres en el papel de beneficiarias de transferencia de renta, tal y como es mencionado por algunos participantes de los grupos focales como una de las causas de la pobreza, es que debe ser un elemento que deber considerarse en las políticas de creación de empleo.

Se evidencia que a las cuestiones presentes en la vida en condiciones de pobreza, se suman los elementos simbólicos. Sobre la evidencia subjetiva, no tener y no ser son dos caras de la pobreza, que además de expresar un orden socioeconómico (cuantitativo, material), también manifiesta una esfera política (cualitativa y material) (Demo, 2001), por tanto, simbólica. Esta doble dimensión también es evidenciada por Accorssi (2011), que revela que la pobreza se conceptualiza a partir de dos aspectos centrales: desde el punto de vista socioeconómico (relacionado con la falta) y desde el punto de vista moral (relacionado con elementos positivos como la fuerza y la riqueza espiritual). En la perspectiva moral, ante la pobreza material, lo que importa es tener tradiciones, valores, una religión, felicidad, autoestima, aspecto evidenciado en esta investigación cuando los sujetos participantes de forma recurrente reivindican para sí la existencia de una dignidad en la pobreza.

Se destaca que los sujetos en condiciones de pobreza, al no reconocerse como pobres, denuncian los estigmas sociales a los que están expuestos. Es necesario que las políticas públicas consideren las redes de culpabilización de los individuos, que nutren el imaginario social de visiones del pobre como alguien acomodado, vago, incapaz de administrar sus recursos y violento (Sprandel, 2006). Finalmente, valorar a los ricos por su capacidad de triunfar en el sistema capitalista y culpar a los pobres por su fracaso son estrategias ideológicas sutiles de legitimación de la exclusión (Guareschi, 2001), que están al servicio del mantenimiento de las inequidades. Se trata de la articulación entre los aspectos materiales y las cuestiones simbólicas asociadas a la pobreza, cuando el acceso precario a los bienes y a los servicios se presenta como justificación en un plano moral y cultural, como el que perpetúa las desigualdades. Por un lado, estarían los sujetos sin poder, expropiados de la capacidad de intervenir sobre la propia realidad y, por otro, aquellos que aseguran la conservación del statu quo a través de redes ideológicas que mantienen la sumisión y la resignación (Góis, 2008).

La existencia de redes de apoyo a las clases populares permite que se potencialicen importantes estrategias de superación de las adversidades presentes en la vida en condiciones de pobreza. La importancia dada al poyo ofrecido por la Iglesia y por la familia son elementos relevantes. Valla (2000) afirma que, en los grupos religiosos, los sujetos encuentran apoyo y también una forma de explicación de las dificultades materiales por las que pasan, así como una posibilidad de resignificación de la vida, se sienten capaces de interferir en su destino y de encontrarle más sentido a la vida. La familia representa un importante factor de protección para los sujetos en situación de vulnerabilidad, lo que ratifica la idea de que la referencia de las políticas públicas en este seguimiento es de gran relevancia (Carvalho, 2010; Morais, Koller, \& Raffaelli, 2012). El reconocimiento de que el territorio congrega modos de vida comunitarios y redes de relaciones singulares, puede aportar, todavía, elementos para pensar en las especificidades de las configuraciones familiares e interacciones entre vecinos que dan los indicativos de las diferentes referencias a la familia y a los vecinos como apoyo en el contexto urbano y en el contexto rural. Hay que considerar que las dinámicas comunitarias en el contexto rural se caracterizan por la personalidad 
que marcan las relaciones entre familias, vecindario y permean todas las prácticas vividas concretamente en el territorio (Dantas \& Oliveira, 2014). La baja referencia a los servicios públicos como espacio de apoyo formal puede estar asociada a las deficiencias en la vinculación de las personas con las instituciones, así como a la poca efectividad en la inserción en el tejido comunitario, lo que hace que las políticas sean referenciadas como locales de obtención de recursos y realización de servicios, pero no como fuente de amparo.

En Brasil, las políticas de asistencia social se destacan como punto de referencia para la intervención (Ministério do Desenvolvimento Social e Combate à Fome, 2004) y, según los resultados de este estudio, se evidencia como un locus que debe valorarse, una vez que surge como elemento protector frente a las adversidades provenientes de los contextos de pobreza. Un mayor acercamiento de las políticas públicas a las familias, a partir de una intervención articulada con el territorio comunitario, puede potenciar el establecimiento de redes comunitarias (Goncalvez de Freitas \& Montero, 2003) y constituirse en elemento crucial de afrontamiento de la pobreza y el reconocimiento y apropiación de las políticas públicas como espacio protector, superando, de esta forma, la baja referencia a las políticas públicas como fuente de apoyo y locus de control social.

El desarrollo de intervenciones colectivas que fortalezcan procesos de concientización y de lectura crítica de la realidad, aliadas al establecimiento de acciones comunitarias en el territorio de acción de las políticas públicas, puede contribuir al cambio de tal escenario. La psicología comunitaria, al inclinarse hacia expresiones subjetivas de pobreza, contribuye al fortalecimiento de la actuación de las políticas públicas y de las redes comunitarias de apoyo. Parte, por tanto, de la necesidad de superar una cotidianidad opresora, marcada por la apatía, por el descrédito en la comprensión de sí como alguien capaz de transformar la propia realidad y a merced de las cuestiones financieras y políticas. El fortalecimiento, en la perspectiva de la psicología comunitaria, demarca la capacidad de los miembros de la comunidad de ser capaces de desarrollar conjuntamente habilidades y recur- sos para tener control sobre sus vidas (Montero, 2006), a través de metodologías participativas y con énfasis en los procesos grupales y de articulación comunitaria.

Las políticas públicas, en especial las de transferencia de renta, alineadas a mejoras en el acceso a educación y salud, tienen gran poder de establecer un cambio de vida de la población en condiciones de pobreza, al anclar su actuación en el énfasis en las redes de apoyo locales, resaltando la familia, la religión y las relaciones entre los vecinos. Tal articulación se constituye como importante estrategia para enfrentar la pobreza. Se concluye también que el análisis e intervención en contextos de pobreza deben considerarla en sus aspectos materiales y psicosociales, dando relevancia a los sufrimientos y opresiones que se producen y perpetúan en el plano simbólico, lo que respalda la necesidad de las contribuciones de la psicología comunitaria en las políticas públicas. Se destaca también la importancia del fortalecimiento de las redes de apoyo establecidas en el contexto comunitario y que el cambio social se confirma cuando a los sujetos de les ofrecen espacios de movilización y articulación, inclusive considerando sus especificidades, sus modos singulares de representación y significación de la realidad. El desarrollo de proyectos, de investigaciones y de intervención que contribuyan en este sentido es de gran importancia.

Los resultados presentados apuntan, también, a la necesidad de desarrollar investigaciones que evalúen el impacto de cada una de las políticas en lo que concierne al afrontamiento de la pobreza, así como, su integración. Cabe resaltar, también, la importancia del desarrollo de estudios que destaquen la dimensión psicosocial de la pobreza, explorando prejuicios en relación con los pobres, las redes de culpabilización y fragilización de estos, la importancia de las redes de apoyo social en el afrontamiento de este flagelo.

\section{Referencias}

Accorssi, A. (2011). Materializações do pensamento social sobre a pobreza (Tesis de doctorado). Pontifícia Universidade Católica do Rio Grande do Sul, Porto Alegre. 
Accorssi, A., Scarparo, H., \& Guareschi, P. (2012). O conceito de pobreza: uma reflexão sobre os interesses do conhecimento. Psicologia Argumento, 30(71), 651-658.

Alfaro, J. (junio, 2013). Psicología comunitaria y políticas sociales: institucionalidad y dinámicas de actores. Global Journal of Community Psychology Practice, 4(2). Recuperado de: http://www.gjcpp. org/pdfs/alfaro-v4i2-20130613.pdf

Alves, J. E. D., \& Cavenagui, S. (2013). O Programa Bolsa Família e as taxas de fecundidade no Brasil. En T. Campello \& M. Neri (Orgs.), Programa Bolsa Família: uma década de inclusão e cidadania (pp. 233-246). Brasília: Ipea.

Bardin, L. (2011). Análise de conteúdo (3. a ed.). Lisboa: Edições 70.

Brasil, Ministério do Desenvolvimento Social e Combate à Fome. (2004). Política nacional de assistência social. Brasília: Autor. Recuperado de www.mds. gov.br/cnas/politicae-nobs/pnas.pdf/download

Brasil, Decreto 7.492 de 2011 [Institui o Plano Brasil Sem Miséria]. Junio 2 de 2011. Recuperado de: http://www.mds.gov.br/webarquivos/legislação/ brasil_sem_

miseria/decreto-no-7492.pdf

Camargo, C., Curralero, C., Licio, E., \& Mostafa, J. (2013). Perfil socioeconômico dos beneficiários do Programa Bolsa Família: o que o cadastro único revela? En T. Campello \& M. Neri (Orgs.), Programa Bolsa Família: uma década de inclusão e cidadania (pp. 156-177). Brasília: Ipea.

Carvalho, V. C., \& Serrano-Garcia, I. (marzo, 2008). ¿Deben participar los psicólogos y psicólogas en política pública?: una mirada desde la psicología social-comunitaria. Psicología para la América Latina, 12. Recuperado de: http://www.psicolatina. org/12/participar.html

Carvalho, M. C. B. (2010). Famílias: conversas sobre políticas públicas e práticas. En L. Trad (Org.), Família contemporânea e saúde: significados, práticas e políticas públicas (pp. 67-87). Rio de Janeiro: Editora Fiocruz.

Castel, R. (2006). Classes sociais, desigualdades sociais, exclusão social. En C. Balsa, L. W. Boneti \& M. Soulet (Orgs.), Conceitos e dimensões da pobreza e da exclusão social: uma abordagem transnacional (pp. 63-77). Ijuí: Ed. Unijui.

Cidade, E., Moura Jr., J., \& Ximenes, V. (2012). Implicações psicológicas da pobreza na vida do povo latino-americano. Psicologia Argumento, 30(68), 87-98.

Dantas, C., \& Oliveira, I. F. (2014). As cidades pequenas como território da ação do psicólogo: desafios teóricos e metodológicos. En M. Dimenstein \& J. F. Leite (Orgs.), Psicologia em pesquisa: cenários de práticas e criações (pp. 93-117). Natal: EDUFRN.

Demo, P. (2001). Pobreza política (6. ${ }^{\mathrm{a}}$ ed.). Campinas: Autores Associados.

Fernandes, J. H. P. (2014). Acesso à educação e combate à desigualdade: o papel da educação no âmbito do plano Brasil sem miséria. En O Brasil sem miséria (pp. 543-561). Brasília: Ministério do Desenvolvimento Social e Combate à Fome.

Góis, C. (2008). Saúde comunitária: pensar e fazer. São Paulo: Editora HUCITEC.

Goncalvez de Freitas, M., \& Montero, M. (2003). Las redes comunitarias. En M. Montero (Ed.), Teoria y practica de la psicologia comunitária: la tensión entre comunidad y sociedad (pp. 173-201). Buenos Aires: Paidós.

Guareschi, P. (2009). Pressupostos epistemológicos implícitos no conceito de libertação. En R. Guzzo \& F. Lacerda, Jr. (Orgs.), Psicologia social para a América Latina: o resgate da psicologia da libertação (pp. 4964). Campinas, SP: Editora Alínea.

Guareschi, P. (2001). Pressupostos psicossociais da exclusão: competitividade e culpabilização. En B. Sawaia (Org.), As artimanhas da exclusão: análise psicossocial e ética da desigualdade social (9. ${ }^{\mathrm{a}} \mathrm{ed}$. , pp. 141-156). Petrópolis: Vozes.

Gutiérrez, G. (2001). Globalización, caos y sujeto en América Latina: el impacto del neoliberalismo y las alternativas. San José: DEI.

Instituto Brasileiro de Geografia e Estatística. (2011). Censo Demográfico 2010. Brasília: Ministério do Planejamento, Orçamento e Gestão.

Instituto de Pesquisa e Estratégia Econômica do Brasil. (2012). Perfil municipal de fortaleza. Tema VIII: o mapa da extrema pobreza (Informe N. 43). Fortaleza: Autor. Recuperado de: http://www.ipece. ce.gov.br/publicacoes/ipece-informe/

Ipece_Informe_43_05_novembro_2012.pdf 
Instituto de Pesquisa e Estratégia Econômica do Brasil. (2014). Perfil básico municipal: Apuiarés. Brasília: Autor. Recuperado de: http://www.ipece.ce.gov.br/ publicacoes/perfil_basico/pbm-2014/Apuiares.pdf

Lane, S. (2002). Histórico e fundamentos da psicología comunitária no Brasil. En R. Campos (Org.), Psicologia social comunitária:da solidariedade à autonomía (pp. 17-34). Petrópolis: Vozes.

Montero, M. (2006).Teoría y práctica de la psicología comunitaria: la tensión entre comunidad y sociedad. Buenos Aires: Paidós.

Montero, M., \& Serrano-García, I. (2011). Una introducción a la psicología comunitaria en América Latina. En M. Montero \& I. Serrano-García (Orgs.), Historias de la psicología comunitaria en América Latina: participación y transformación (pp. 23-40). Buenos Aires: Paidós.

Montero, M., \& Soon, C. (2009). About liberation and psychology: An introduction. En M. Montero \& C. Soon (Eds.), Psychology of liberation: Theory and Applications (pp. 1-10). Nueva York: Springer.

Morais, N. A., Koller, S. H., \& Raffaelli, M. (2012). Rede de apoio, eventos estressores e mau ajustamento na vida de crianças e adolescentes em situação de vulnerabilidade social. Universitas Psychologica, 11(3), 779-791.

Moura, J., Jr., Cidade, E. C., Ximenes, V. M, \& Sarriera, J. C. (2014). Concepções de pobreza: um convite à discussão psicossocial. Temas em Psicologia, 22(2), 341-352.

Programa de las Naciones Unidas para el Desarrollo. (2014). Relatório do Desenvolvimento Humano 2014. Sustentar o Progresso Humano: Reduzir as Vulnerabilidades e Reforçar a Resiliência (Informe RDH2014). Nueva York: Autor. Recuperado de: http://www. pnud.org.br/arquivos/RDH2014pt.pdf

Rêgo, W., \& Pinzani, A. (2013). Vozes do Bolsa Família: autonomia, dinheiro e cidadania. São Paulo: Ed. Unesp.

Rodríguez, A. (2012). Aportes de la psicología comunitaria al campo de las políticas públicas sociales: el caso del Uruguay. En J. Alfarro, A. Sánchez \& A. Zambrani (Comps.), Psicología comunitaria y políticas sociales (pp. 111-146). Buenos Aires: Paidós.

Sen, A. (2010). Desenvolvimento como liberdade. São Paulo: Companhia das Letras.
Silva, A. P, Brandão, A., \& Dalt, S. (2009). Educação e pobreza: o impacto das condicionalidades do programa bolsa família. Revista contemporânea de Educação, 4(8), 296-313.

Souza, P., \& Osório, R. (2013). O perfil da pobreza no Brasil e suas mudanças entre 2003 e 2011. En T. Campello \& M. Neri (Orgs.), Programa Bolsa Família: uma década de inclusão e cidadania (pp. 139-156). Brasília: Ipea.

Sprandel, M. (2006). Nossos pobres ou nosso povo? Contribuição para o debate sobre políticas sociais no Brasil. Inclusão Social, 1(2), 83-87.

Sposati, A. (2003). Regulação social tardia: característica das políticas sociais latino-americanas na passagem entre o segundo e o terceiro milênio. Intervenção Social, 1(27), 63-88.

Torossian, S. D., \& Rivero, N. E. (2013). Políticas públicas e modos de viver - a produção de sentidos sobre a vulnerabilidade. En L. R. Cruz \& N. Guareschi (Orgs.), Políticas públicas e assistência social: diálogo com as práticas psicológicas. (pp. 56-69). Petrópolis, RJ: Vozes.

Valla, V. (2000). Redes sociais, poder e saúde à luz das classes populares numa conjuntura de crise. Interface: Comunicação, Saúde, Educação, 4(7), 37-56.

Vygotsky, L. (2000). Manuscrito de 1929 (A. Marenitch, Trad.). Educação e Sociedade, 21(71), 21-44.

Wiesenfeld, E. (2011). Community social psychology in Latin America: Myths, dilemmas and challenges. En F. H. E. Almeida, G. Hinojosa, O. Soto, G. Inguanzo, M. E. Sánchez \& C. Cuétara Priede (Eds.), International community psychology: Community approaches to contemporarysocial problems (Vol.1, pp. 95-122). Puebla, México: Universidad Iberoamericana Puebla.

Wiesenfeld, E., \& Sánchez, E. (2012) Participación, pobreza y políticas públicas: 3P que desafían la psicología ambiental comunitaria (el caso de los Consejos Comunales de Venezuela). Psychosocial Intervention, 21(3),225-243.

Zavaleta, R. (mayo, 2007). The ability to go about without shame: A proposal for internationally comparable indicators of shame and humiliation (OPHI Working Paper Series N. ${ }^{\circ}$ ). Oxford: Oxford Poverty \& Human Development Iniciative. Recuperado de: http://www.ophi.org.uk/working-paper-number-03 